\title{
Triple diffusive convection in Oldroyd-B liquid
}

\author{
Sameena Tarannum ${ }^{1}$ and S. Pranesh ${ }^{2}$ \\ ${ }^{1}$ Department of Professional Studies, Christ University, Bangalore, India. \\ ${ }^{2}$ Department of Mathematics, Christ University, Bangalore, India.
}

\begin{abstract}
A linear stability analysis is carried out for triple diffusive convection in Oldroyd-B liquid. The expressions of Rayleigh number for stationary and oscillatory convection has been obtained. The neutral curves for oscillatory mode for different values of stress relaxation parameter, strain retardation parameter, solute Rayleigh numbers, ratio of diffusivity of solutes and heat diffusivity and Prandtl number has been examined. It is found that the oscillatory triple diffusive convection sets in earlier in system with Oldroyd-B liquid as compared to Newtonian liquid system.
\end{abstract}

Keywords: Triple diffusive convection, viscoelastic liquid, Oldroyd-B liquid, oscillatory convection.

\section{Introduction}

In the standard Rayleigh Bénard problem, the instability is driven by a density difference caused by the temperature difference between the upper and lower plates bounding the liquid. If the liquid additionally has salt dissolved in it, then there are potentially two destabilizing sources for the density difference, the temperature field and salt field. The solution behaviour in the double-diffusive convection problem is more interesting than that of the single component situation in so much as new instability phenomena may occur which is not present in the classical Bénard problem. When temperature and two or more component agencies, or, temperature and two more different salts of different diffusivity are present then the physical and mathematical situation becomes increasingly richer. Very interesting results in triply diffusive convection have been obtained by Pearlstein et al. [1]. The results of Pearlstein et al., are remarkable. They demonstrate that for triple diffusive convection linear instability can occur in discrete sections of the Rayleigh number domain with the liquid being linearly stable in a region in between the linear instability ones. This is because for certain parameters the neutral curve has a finite isolated oscillatory instability curve lying below the usual unbounded stationary convection one.

The problem under investigation also has many physical applications in solidification of alloys, the materials processing, underground of chemical pollutants and petroleum reservoirs. The problems of triple diffusive convection are studied by Pearlstein et al. [1], Lopez et al. [2], Rudraiah and Vortmeyer [3], Poulikakos [4], Suresh Chand [5 - 7], Sumithra [8] andRionero [9].

The study of non-Newtonian liquids has gained tremendous interest because of its usage as a working media in many engineering and industrial applications. Viscoelastic liquids which exhibit both solid and liquid properties have applications in such diverse fields as geothermal energy modeling, material processing, thermal insulation material, cooling of electronic devices, transport of chemical substances, crystal growth, injection moulding and solar receivers. Other applications are found in the petroleum industry, chemical and nuclear industries, geophysics, bioengineering and so on.

The rheological equation for viscoelastic liquid usually involves either one or two relaxation times. They possess both elasticity (associated with solids) and viscosity (associated with liquids) which leads to unique instability patterns such as over stability that is not predicted or observed in Newtonian liquids. Hence, Rayleigh-Bénard convection in a thin rectangular layer of viscoelastic liquid heated from below has been the focus of many studies over the past few decades. Many researchers like Riahi [10], Siddeshwar et al. [11], Narayana et al. [12, 13], Malashetty et al. [14] and recently Bhadauria and Kiran [15] have considered viscoelastic liquids.

With the motivation directed by the applications, the main aim of this paper is to study triple-diffusive convection in Oldroyd-B liquids.

\section{Mathematical Formulation}

Consider a layer of Oldroyd-B liquid confined between two infinite horizontal surfaces separated by a distance $\mathrm{d}$ apart. The uniform is directed along the $\mathrm{z}$-axis. A Cartesian system is taken with origin in the lower boundary and z-axis vertically upward (see figure 1). Let $\Delta \mathrm{T}, \Delta \mathrm{S}_{1}$ and $\Delta \mathrm{S}_{2}$ be the differences in temperature and solute concentrations, respectively of the liquid between the lower and upper plates. Appropriate single-phase heat and two-phase solute transport equations are chosen with effective heat capacity ratios and effective thermal diffusivity. 


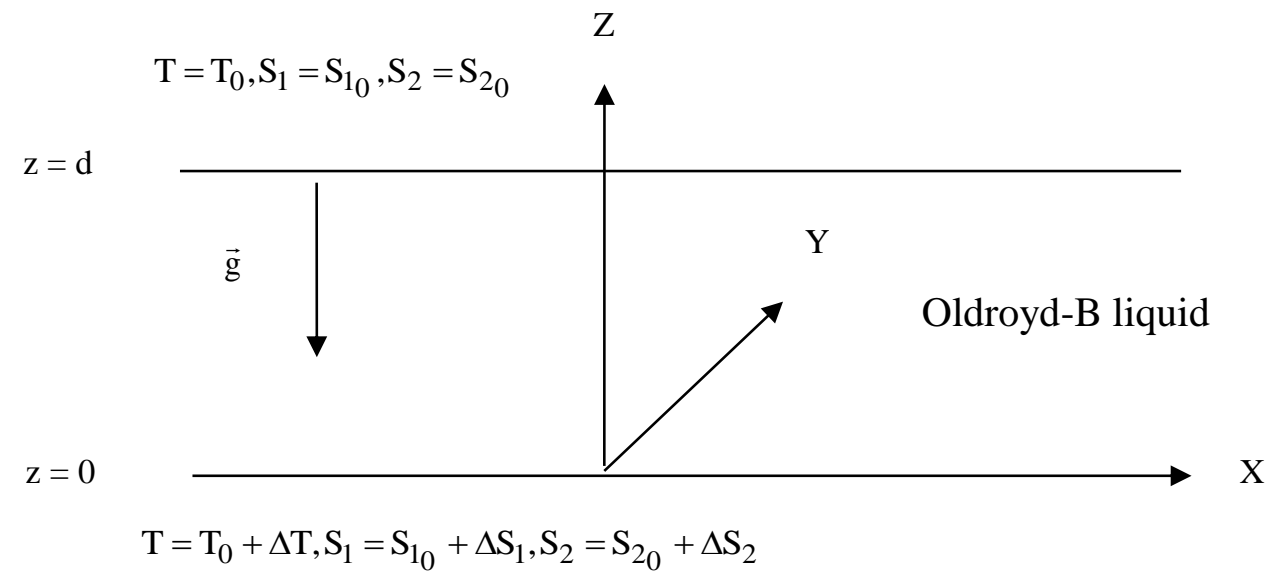

Figure 1: Physical configuration

The governing equations in Oldroyd-B liquid in triple diffusive convection are:

$\nabla \cdot \vec{q}=0$

$\rho_{\mathrm{o}} \frac{\partial \overrightarrow{\mathrm{q}}}{\partial \mathrm{t}}=-\nabla \mathrm{p}+\rho \overrightarrow{\mathrm{g}}+\nabla \cdot \tau^{\prime}$,

$\left(1+\lambda_{1} \frac{\partial}{\partial \mathrm{t}}\right) \tau^{\prime}=\mu\left(1+\lambda_{2} \frac{\partial}{\partial \mathrm{t}}\right)\left(\nabla \overrightarrow{\mathrm{q}}+\nabla \overrightarrow{\mathrm{q}}^{\mathrm{tr}}\right)$,

$\frac{\partial \mathrm{T}}{\partial \mathrm{t}}+(\overrightarrow{\mathrm{q}} \cdot \nabla) \mathrm{T}=\chi \nabla^{2} \mathrm{~T}$,

$\frac{\partial \mathrm{S}_{1}}{\partial \mathrm{t}}+(\overrightarrow{\mathrm{q}} \cdot \nabla) \mathrm{S}_{1}=\chi_{\mathrm{S} 1} \nabla^{2} \mathrm{~S}_{1}$,

$\frac{\partial \mathrm{S}_{2}}{\partial \mathrm{t}}+(\overrightarrow{\mathrm{q}} . \nabla) \mathrm{S}_{2}=\chi_{\mathrm{S} 2} \nabla^{2} \mathrm{~S}_{2}$,

$\rho=\rho_{0}\left[1-\alpha_{t}\left(T-T_{0}\right)+\alpha_{S 1}\left(S_{1}-S_{10}\right)+\alpha_{S 2}\left(S_{2}-S_{2_{0}}\right)\right]$.

where, $\vec{q}$ is velocity, $p$ is pressure, $\rho_{0}$ is the constant density, $\rho$ is density, $\vec{g}$ is gravitational force, $\tau$ ' is the stress tensor, $\lambda_{1}$ is the stress relaxation time, $\lambda_{2}$ is the strain retardation time, $\mu$ is viscosity, $\mathrm{T}$ is temperature, $S_{1}$ is solute $1, S_{2}$ is solute2, $\chi$ is the thermal diffusivity, $\chi_{\mathrm{S} 1}$ is solute1 diffusivity, $\chi_{\mathrm{S} 2}$ is solute2 diffusivity, $\alpha_{\mathrm{t}}$ is coefficient of thermal expansion to determine how fast the density decreases with temperature, $\alpha_{\mathrm{S} 1}$ is the coefficient of solute1 expansion and $\alpha_{\mathrm{S} 2}$ is the coefficient of solute2 expansion.

It is to be noted that the convective acceleration term ( $\overrightarrow{\mathrm{q}} \cdot \nabla) \overrightarrow{\mathrm{q}}$ has been dropped in equation (2) in comparison with the heat advection term $(\overrightarrow{\mathrm{q}} . \nabla) \mathrm{T}$ vowing to the assumption that thermally induced instabilities dominate hydrodynamic instabilities. This also implies that the present analysis is restricted to small scale convective motions.

Operating divergence on equation (3) and using (2), we get,

$\left(1+\lambda_{1} \frac{\partial}{\partial \mathrm{t}}\right)\left(\rho_{\mathrm{o}} \frac{\partial \overrightarrow{\mathrm{q}}}{\partial \mathrm{t}}+\nabla \mathrm{p}+\rho \mathrm{gk}\right)=\mu\left(1+\lambda_{2} \frac{\partial}{\partial \mathrm{t}}\right) \nabla^{2} \overrightarrow{\mathrm{q}}$.

Equations (1) - (7) are solved for free-free velocity, isothermal and isoconcentration boundary conditions:

$\mathrm{w}=\frac{\partial^{2} \mathrm{w}}{\partial \mathrm{z}^{2}}=\mathrm{T}=\mathrm{S}_{1}=\mathrm{S}_{2}=0$ at $\mathrm{z}=0,1$.

The basic state of the liquid is assumed to be quiescent and is described by:

$\overrightarrow{\mathrm{q}}_{\mathrm{b}}=(0,0,0), \mathrm{p}=\mathrm{p}_{\mathrm{b}}(\mathrm{z}), \rho=\rho_{\mathrm{b}}(\mathrm{z}), \mathrm{T}=\mathrm{T}_{\mathrm{b}}(\mathrm{z}), \mathrm{S}_{1}=\mathrm{S}_{1 \mathrm{~b}}(\mathrm{z}), \mathrm{S}_{2}=\mathrm{S}_{2_{\mathrm{b}}}(\mathrm{z})$,

where the subscript ' $b$ ' denotes the basic state. Substituting equation (8) into equations (1) to (7),we get, 


$$
\left.\begin{array}{l}
\frac{d p_{b}}{d z}+\rho_{b} g=0, \\
\frac{\partial^{2} T_{b}}{\partial z^{2}}=0, \\
\frac{\partial^{2} S_{1 b}}{\partial z^{2}}=0, \\
\frac{\partial^{2} S_{2 b}}{\partial z^{2}}=0, \\
\rho_{b}=\rho_{o}\left[1-\alpha_{t}\left(T_{b}-T_{0}\right)+\alpha_{S 1}\left(S_{1 b}-S_{1_{0}}\right)+\alpha_{S 2}\left(S_{2 b}-S_{2_{0}}\right)\right] .
\end{array}\right\}
$$

The stability of the basic state is analysed by introducing the following perturbation:

$$
\left.\begin{array}{l}
\overrightarrow{\mathrm{q}}=\overrightarrow{\mathrm{q}}_{\mathrm{b}}+\overrightarrow{\mathrm{q}}^{\prime}, \mathrm{p}=\mathrm{p}_{\mathrm{b}}+\mathrm{p}^{\prime}, \rho=\rho_{\mathrm{b}}+\rho^{\prime}, \\
\mathrm{T}=\mathrm{T}_{\mathrm{b}}+\mathrm{T}^{\prime}, \mathrm{S}_{1}=\mathrm{S}_{1_{\mathrm{b}}}+\mathrm{S}_{1}^{\prime}, \mathrm{S}_{2}=\mathrm{S}_{2_{\mathrm{b}}}+\mathrm{S}_{2}^{\prime}
\end{array}\right\},
$$

where, the prime indicates that the quantities are infinitesimal perturbations.

Substituting equation (12) in equations (1) to (7), using basic state solutions (11) and by nondimensionlisingusing the following definitions:

$$
\left.\begin{array}{l}
\left(\mathrm{x}^{*}, \mathrm{y}^{*}, \mathrm{z}^{*}\right)=\left(\frac{\mathrm{x}^{\prime}}{\mathrm{d}}, \frac{\mathrm{y}^{\prime}}{\mathrm{d}}, \frac{\mathrm{z}^{\prime}}{\mathrm{d}}\right), \mathrm{t}^{*}=\frac{\mathrm{t}^{\prime}}{\mathrm{d}^{2} / \chi}, \mathrm{q}^{*}=\frac{\mathrm{q}^{\prime}}{\chi / \mathrm{d}}, \\
\mathrm{p}^{*}=\frac{\mathrm{d}^{2}}{\mu \chi} \mathrm{p}^{\prime}, \theta=\frac{\mathrm{T}^{\prime}}{\Delta \mathrm{T}}, \phi_{\mathrm{S} 1}=\frac{\mathrm{S}_{1}^{\prime}}{\Delta \mathrm{S}_{1}}, \phi_{\mathrm{S} 2}=\frac{\mathrm{S}_{2}^{\prime}}{\Delta \mathrm{S}_{2}}
\end{array}\right\},
$$

We obtain the following dimensionless equations by eliminating the pressure term and also by introducing stream function $\psi$ (after dropping the asterisk):

$$
\begin{aligned}
& \left(1+\Lambda_{1} \frac{\partial}{\partial \mathrm{t}}\right)\left[\frac{1}{\operatorname{Pr}} \frac{\partial}{\partial \mathrm{t}}\left(\nabla^{2} \psi\right)-\left(-\mathrm{Ra} \frac{\partial \theta}{\partial \mathrm{x}}+\mathrm{R}_{\mathrm{S} 1} \frac{\partial \phi_{\mathrm{S} 1}}{\partial \mathrm{x}}+\mathrm{R}_{\mathrm{S} 2} \frac{\partial \phi_{\mathrm{S} 2}}{\partial \mathrm{x}}\right)\right]=\left(1+\Lambda_{2} \frac{\partial}{\partial \mathrm{t}}\right) \nabla^{4} \psi, \\
& \frac{\partial \theta}{\partial \mathrm{t}}+\frac{\partial \psi}{\partial \mathrm{x}}=\nabla^{2} \theta, \\
& \frac{\partial \phi_{\mathrm{S} 1}}{\partial \mathrm{t}}+\frac{\partial \psi}{\partial \mathrm{x}}=\tau_{1} \nabla^{2} \phi_{\mathrm{S} 1}, \\
& \frac{\partial \phi_{\mathrm{S} 2}}{\partial \mathrm{t}}+\frac{\partial \psi}{\partial \mathrm{x}}=\tau_{2} \nabla^{2} \phi_{\mathrm{S} 2},
\end{aligned}
$$

where, $\Lambda_{1}=\frac{\chi \lambda_{1}}{\mathrm{~d}^{2}}$ is stress relaxation parameter or Deborah number,

$\Lambda_{2}=\frac{\chi \lambda_{2}}{\mathrm{~d}^{2}}$ is strain retardation parameter,

$\operatorname{Pr}=\frac{\mu}{\rho_{0} \chi}$ is Prandtl number,

$\mathrm{Ra}=\frac{\rho_{\mathrm{o}} \alpha_{\mathrm{t}} \mathrm{g} \Delta \mathrm{Td}^{3}}{\mu \chi}$ is Rayleigh number,

$\mathrm{R}_{\mathrm{S} 1}=\frac{\rho_{\mathrm{o}} \alpha_{\mathrm{S} 1} \mathrm{~g} \Delta \mathrm{S}_{1} \mathrm{~d}^{3}}{\mu \chi}$ is solute1 Rayleigh number,

$\mathrm{R}_{\mathrm{S} 2}=\frac{\rho_{\mathrm{o}} \alpha_{\mathrm{S} 2} \mathrm{~g} \Delta \mathrm{S}_{2} \mathrm{~d}^{3}}{\mu \chi}$ is solute2 Rayleigh number,

$\tau_{1}=\frac{\chi_{\mathrm{S} 1}}{\chi}$ is ratio of diffusivity of solute 1 and heat diffusivity and 
$\tau_{2}=\frac{\chi_{\mathrm{S} 2}}{\chi}$ is ratio of diffusivity of solute 2 and heat diffusivity.

Eliminating $\theta, \phi_{\mathrm{S} 1}$ and $\phi_{\mathrm{S} 2}$ from equations (14) - (17), we get the equation of $\psi$ in the form of:

$\left[\mathrm{X}_{4} \mathrm{X}_{3} \mathrm{X}_{2} \mathrm{X}_{1}-\left(\mathrm{RaX}_{4} \mathrm{X}_{3}-\mathrm{R}_{\mathrm{S}} \mathrm{X}_{4} \mathrm{X}_{2}-\mathrm{R}_{\mathrm{C}} \mathrm{X}_{3} \mathrm{X}_{2}\right) \frac{\partial^{2}}{\partial \mathrm{x}^{2}}\right] \psi=0$

where, $X_{1}=\left[\left(1+\Lambda_{1} \frac{\partial}{\partial t}\right) \frac{1}{\operatorname{Pr}} \frac{\partial}{\partial t}-\left(1+\Lambda_{2} \frac{\partial}{\partial t}\right) \nabla^{2}\right]$,

$\mathrm{X}_{2}=\left(\frac{\partial}{\partial \mathrm{t}}-\nabla^{2}\right)$,

$\mathrm{X}_{3}=\left(\frac{\partial}{\partial \mathrm{t}}-\tau_{1} \nabla^{2}\right)$ and

$\mathrm{X}_{4}=\left(\frac{\partial}{\partial \mathrm{t}}-\tau_{2} \nabla^{2}\right)$.

In dimensionless form, the boundary conditions (9) for solving equation (18) are obtained in the form:

$\psi=\frac{\partial^{2} \psi}{\partial \mathbf{z}^{2}}=\frac{\partial^{4} \psi}{\partial \mathbf{z}^{4}}=\frac{\partial^{6} \psi}{\partial \mathbf{z}^{6}}=\frac{\partial^{8} \psi}{\partial \mathrm{z}^{8}}=\frac{\partial^{10} \psi}{\partial \mathbf{z}^{10}}=\frac{\partial^{12} \psi}{\partial \mathbf{z}^{12}}=0$ at $\mathrm{z}=0,1$.

At the lowest mode, let

$\psi=\psi_{0} \sin (\pi \mathrm{z}) \exp [\mathrm{i}(\mathrm{lx}+\mathrm{my})+\sigma \mathrm{t}]$.

Substituting equation (20) in equation (18), we get,

$\mathrm{Ra}=\frac{\left\{\begin{array}{l}\left(\sigma+\mathrm{k}^{2}\right)\left(\sigma+\tau_{1} \mathrm{k}^{2}\right)\left(\sigma+\tau_{2} \mathrm{k}^{2}\right)\left[\left(1+\Lambda_{1} \sigma\right) \frac{\sigma}{\mathrm{Pr}}+\left(1+\Lambda_{2} \sigma\right) \mathrm{k}^{2}\right] \mathrm{k}^{2} \\ +\mathrm{R}_{\mathrm{S}}\left(\sigma+\mathrm{k}^{2}\right)\left(\sigma+\tau_{2} \mathrm{k}^{2}\right) \mathrm{a}^{2}+\mathrm{R}_{\mathrm{S} 2}\left(\sigma+\mathrm{k}^{2}\right)\left(\sigma+\tau_{1} \mathrm{k}^{2}\right) \mathrm{a}^{2}\end{array}\right\}}{\left(\sigma+\tau_{1} \mathrm{k}^{2}\right)\left(\sigma+\tau_{2} \mathrm{k}^{2}\right) \mathrm{a}^{2}}$.

\subsection{Stationary convection}

For stationary convection, $\sigma$ in equation (21) must be real and the corresponding Rayleigh number $\mathrm{Ra}^{\mathrm{st}}$ for stationary convection is obtained by putting $\sigma=0$ in equation (21) in the form:

$\mathrm{Ra}^{\mathrm{st}}=\frac{\mathrm{R}_{\mathrm{S} 1}}{\tau_{1}}+\frac{\mathrm{R}_{\mathrm{S} 2}}{\tau_{2}}+\frac{\mathrm{k}^{6}}{\mathrm{a}^{2}}$,

where $\mathrm{k}^{2}=\pi^{2}+\mathrm{a}^{2}$ and $\mathrm{a}^{2}=\mathrm{l}^{2}$.

\subsection{Oscillatory convection}

To study oscillatory motions we put $\sigma=i \omega$, where $\omega \in \mathfrak{R}$, in equation (21), we obtain the expression of Rayleigh number for oscillatory convection as

$\mathrm{Ra}^{\mathrm{oc}}=\frac{\mathrm{R}_{\mathrm{S} 1}\left(\tau_{1} \mathrm{k}^{4}+\omega^{2}\right)}{\left(\tau_{1}^{2} \mathrm{k}^{4}+\omega^{2}\right)}+\frac{\mathrm{R}_{\mathrm{S} 2}\left(\tau_{2} \mathrm{k}^{4}+\omega^{2}\right)}{\left(\tau_{2}^{2} \mathrm{k}^{4}+\omega^{2}\right)}+\frac{\left(1+\Lambda_{1} \Lambda_{2} \omega^{2}\right) \mathrm{k}^{6}}{\left(1+\Lambda_{1}^{2} \omega^{2}\right) \mathrm{a}^{2}}+\frac{\left(\Lambda_{1}-\Lambda_{2}\right) \omega^{2} \mathrm{k}^{4}}{\left(1+\Lambda_{1}^{2} \omega^{2}\right) \mathrm{a}^{2}}-\frac{\omega^{2} \mathrm{k}^{2}}{\mathrm{a}^{2} \operatorname{Pr}}$,

and frequency,

$\mathrm{A} \omega^{6}+\mathrm{B} \omega^{4}+\mathrm{C} \omega^{2}+\mathrm{D}=0$,

where, $\mathrm{A}=\Lambda_{1} \Lambda_{2} \mathrm{k}^{4}+\frac{\Lambda_{1}^{2} \mathrm{k}^{4}}{\operatorname{Pr}}$,

$\mathrm{B}=\left[\begin{array}{l}\mathrm{R}_{\mathrm{S} 1}\left(\tau_{1}-1\right) \mathrm{k}^{2} \mathrm{a}^{2} \Lambda_{1}^{2}+\mathrm{R}_{\mathrm{S} 2}\left(\tau_{2}-1\right) \mathrm{k}^{2} \mathrm{a}^{2} \Lambda_{1}^{2}-\left(\Lambda_{1}-\Lambda_{2}\right) \mathrm{k}^{6} \\ +\left(1+\frac{1}{\operatorname{Pr}}\right) \mathrm{k}^{4}+\left(\frac{\Lambda_{1}^{2}}{\operatorname{Pr}}+\Lambda_{1} \Lambda_{2}\right)\left(\tau_{1}^{2}+\tau_{2}^{2}\right) \mathrm{k}^{8}\end{array}\right]$, 


$$
\begin{aligned}
& \mathrm{C}=\left[\begin{array}{l}
\mathrm{R}_{\mathrm{S} 1}\left(\tau_{1}-1\right) \mathrm{k}^{2} \mathrm{a}^{2}+\mathrm{R}_{\mathrm{S} 2}\left(\tau_{2}-1\right) \mathrm{k}^{2} \mathrm{a}^{2}+\mathrm{R}_{\mathrm{S} 1}\left(\tau_{1}-1\right) \tau_{2}^{2} \mathrm{k}^{6} \mathrm{a}^{2} \Lambda_{1}^{2}+\mathrm{R}_{\mathrm{S} 2}\left(\tau_{2}-1\right) \tau_{1}^{2} \mathrm{k}^{6} \mathrm{a}^{2} \Lambda_{1}^{2} \\
+\left(1+\frac{1}{\operatorname{Pr}}-\Lambda_{1} \mathrm{k}^{2}+\Lambda_{2} \mathrm{k}^{2}\right)\left(\tau_{1}^{2}+\tau_{2}^{2}\right) \mathrm{k}^{8}+\left(\frac{\Lambda_{1}^{2}}{\operatorname{Pr}}+\Lambda_{1} \Lambda_{2}\right) \tau_{1}^{2} \tau_{2}^{2} \mathrm{k}^{12}
\end{array}\right], \\
& \mathrm{D}=\left[\mathrm{R}_{\mathrm{S} 1}\left(\tau_{1}-1\right) \tau_{2}^{2} \mathrm{k}^{6} \mathrm{a}^{2}+\mathrm{R}_{\mathrm{S} 2}\left(\tau_{2}-1\right) \tau_{1}^{2} \mathrm{k}^{6} \mathrm{a}^{2}+\left(1+\frac{1}{\operatorname{Pr}}-\Lambda_{1} \mathrm{k}^{2}+\Lambda_{2} \mathrm{k}^{2}\right) \tau_{1}^{2} \tau_{2}^{2} \mathrm{k}^{12}\right] .
\end{aligned}
$$

\section{Results And Discussion}

The onset of three-component convection in viscoelastic Oldroyd-B liquid heated and salted from below has been investigated using linear theory. Analytical solution for Rayleigh number is obtained using linear theory. The primary objective of this study was to determine the effect of viscoelastic parameters like stress relaxation parameter and strain retardation parameter on the onset of triple diffusive convection. The stationary critical Rayleigh number is found to be independent of the viscoelastic parameters $\Lambda_{1}$ and $\Lambda_{2}$, and this reduces to the classical results of triply diffusive fluid layer (see [1]).

Figure 2 shows the neutral curves for different values of stress relaxation parameter, strain retardation parameter, solute Rayleigh numbers, ratio of diffusivity of solutes and heat diffusivity and Prandtl number. Figure 2(a) depicts the neutral curves for stress relaxation parameter $\Lambda_{1}$. We note that the effect of increase in the stress relaxation parameter, decreases the critical oscillatory Rayleigh number, indicating that the stress relaxation parameter advances the onset of triple diffusive convection, hence destabilises the system. On the other hand, figure 2(b) shows that the increase in strain retardation parameter $\Lambda_{2}$, increases the critical oscillatory Rayleigh number, thus stabilises the system. Further, we observe from figures 2(a) and 2(b) that critical Rayleigh number for stationary mode (Newtonian liquid) is higher than that of oscillatory mode. Since all the curves lie below that of a Newtonian liquid curve, this indicates that the oscillatory convection sets in earlier in system with viscoelastic liquid as compared to Newtonian liquid system.

Figure 2(c) and 2(d) are the neutral curves for different values of solute Rayleigh number1 $R_{\mathrm{S} 1}$ and solute Rayleigh number2 $R_{\mathrm{S} 2}$, respectively. Here we observe that as $R_{\mathrm{S} 1}$ and $R_{\mathrm{S} 2}$ increases, the oscillatory Rayleigh number also increases thus $R_{S 1}$ and $R_{S 2}$ stabilises the system. Since we solute from below, the concentration of the solutes settles at the bottom without disturbing the system and hence the solute Rayleigh numbers enhances the stability of the system. Positive values of $R_{S 1}$ and $R_{S 2}$ are considered and in such a case, one gets positive values of Ra and these signify the assumption of a situation heating from below. The heating expands the liquid at the bottom of the layer, this expands the liquid thereby enhance the motion due to thermal convection, on the other side, the heavier salts at the lower part of the layer have exactly the opposite effect and these act to prevent motion through convective over turning. Thus these two effects are competing each other and hence onset of convection is delayed.

Figure 2(e) and 2(f) are the neutral curves for different values of ratio of diffusivity of solute 1 and heat diffusivity $\tau_{1}$ and ratio of diffusivity of solute 2 and heat diffusivity $\tau_{2}$, respectively. It is observed that increasing the values of $\tau_{1}$ and $\tau_{2}$, decreases oscillatory Rayleigh number, indicating that the ratio of diffusivity of solutes and heat diffusivity advances the onset of triple diffusive convection and hence destabilises the system. This is because, the diffusivity of heat is more than the diffusivity of solutes and therefore, solute gradient augments the onset of convection.Figure 2(g) is the neutral curves for different values of Prandtl number Pr. Here we do not see much significant change, however, as Pr increases, the oscillatory Rayleigh number decreases and advances the onset of triple diffusive convection thus destabilises the system. 


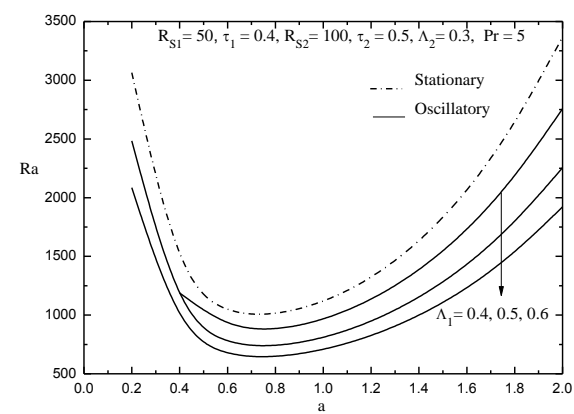

(a)

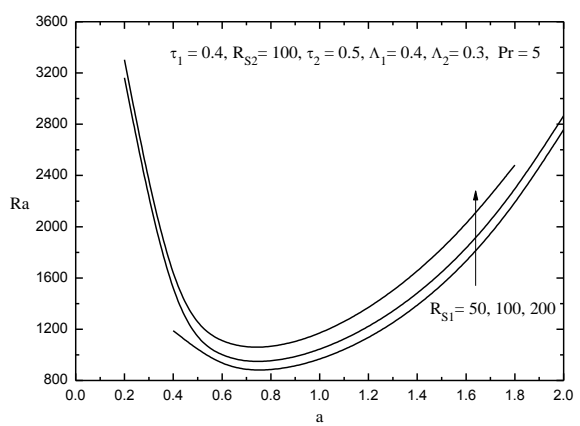

(c)

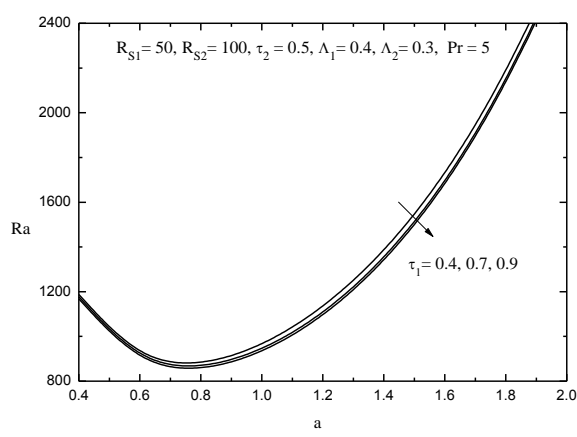

(e)

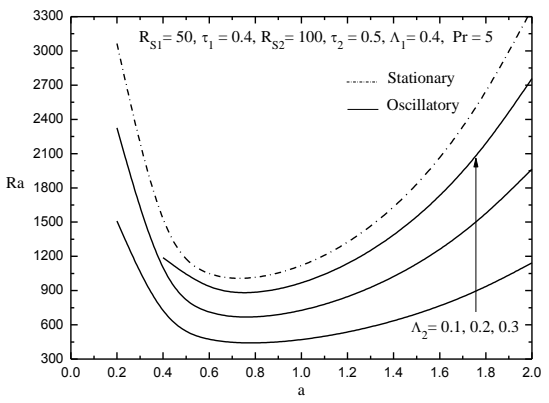

(b)

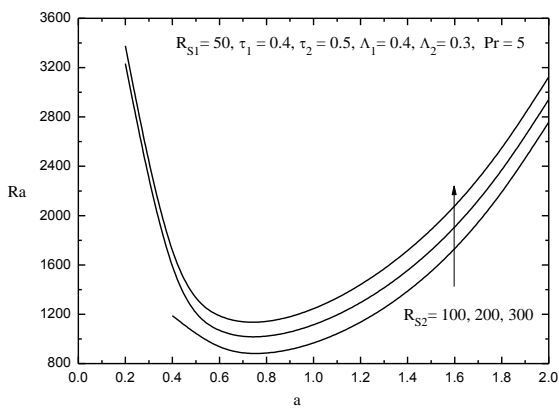

(d)

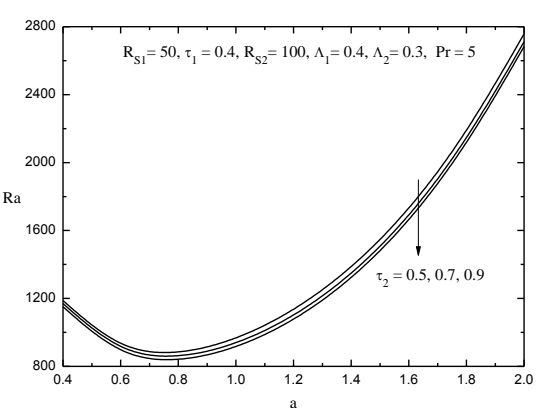

(f)

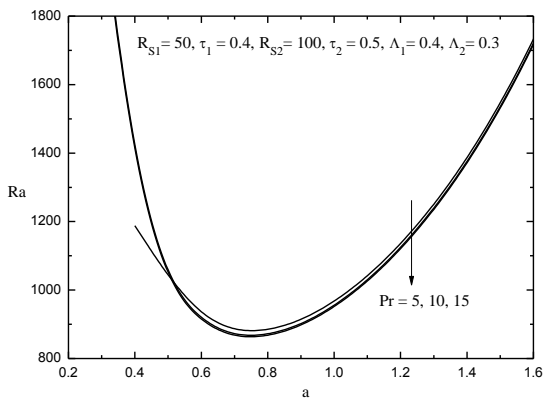

(g)

Figure 2: Neutral curves for oscillatory mode for different values of (a) $\Lambda_{1}$ (b) $\Lambda_{2}$ (c) $R_{\mathrm{S} 1}$ (d) $R_{\mathrm{S} 2}$ (e) $\tau_{1}$ (f) $\tau_{2}$ (g) $\operatorname{Pr}$ 


\section{Conclusion}

Following conclusions are drawn from the present study:

i. Stress relaxation parameter and solute Rayleigh numbers advances the onset of triple diffusive convection.

ii. Strain retardation parameter,ratio of diffusivity of solutes and heat diffusivity and Prandtl number delays the onset of triple diffusive convection.

iii. The oscillatory triple diffusive convection sets in earlier in system with viscoelastic liquid as compared to Newtonian liquid system.

\section{Acknowledgement}

Authors would like to acknowledge management of Christ University for their support in completing the work.

\section{References}

[1] Pearlstein, A. J., et al., 1989, “The onset of convective instability in a triply diffusive fluid layer,” J. Fluid Mech., 202, pp. $443-465$.

[2] Lopez R. A., et al., 1990, "Effect of rigid boundaries on the onset of convective instability in a triply diffusive fluid layer," Phys. Fluids A, 2, pp. 897.

[3] Rudraiah, N., and Vortmeyer, D., 1982, "The influence of permeability and of a third diffusing component upon the onset of convection in a porous medium," Int. J. Heat and mass transfer, 25, pp. 457.

[4] Poulikakos, D., 1985, "The effect of a third diffusing component on the onset of convection in a horizontal porous layer," Phys. Fluids, 28, pp. 3172.

[5] Chand, S., 2012, "Triple-Diffusive convection in a micropolarferrofluid in the presence of rotation," Int. J. Engg. Research and Tech., 1(5), pp. 1-14

[6] Chand, S., 2012, "Triple-diffusive convection in a magnetized ferrofluid with MFD viscosity: A nonlinear stability analysis," IOSR J. Math., 3(5), pp. 20-28.

[7] Chand, S., 2012, "Effect of rotation on triple-diffusive convection in a magnetized ferrofluid with internal angular momentum saturating a porous medium," Applied Mathematical Sciences, 6(65), pp. $3245-3258$.

[8] Sumithra, R., 2012, "Exact solution of triple diffusive marangoniconvection in a composite layer.” Int. J. Engg. Research and Tech., 1(5), pp. 1-13.

[9] Rionero, S., 2013, "Triple diffusive convection in porous media," ActaMech, 224, pp. 447-458.

[10] Riahi, N., 1976, Nonlinear convection in a viscoelasric fluid,” J. Math. Phys. Sci., 26, pp. 211-220.

[11] Siddheshwar, P. G., et al., 2010, "Surface tension driven convection in viscoelastic liquids with thermorheological effect," Int. Comm. Heat and Mass transfer, 38(4), pp. 468-473.

[12] Narayana, M., et al., 2013, "Double diffusive magneto-convection in viscoelastic fluids,” Int. J. Heat and Mass Transfer, 67, pp. 194-201.

[13] Narayana, M., et al., 2013, "Linear and nonlinear stability analysis of binary viscoelastic fluid convection,” Applied Mathematical Modelling, 37, pp. 8162-8178.

[14] Malashetty, M., and Swamy, M., 2010, “The onset of double diffusive convection in a viscoelastic fluid layer," J. Non-Newtonian Fluid Mech., 165, pp. 1129-1138.

[15] Bhadauria, B. S., and Kiran P., 2015, “Chaotic and oscillatory magneto-convection ina binary viscoelastic fluid under g-jitter," Int. J Heat and Mass Transfer, 84, pp. 610-624 